\title{
An unusual new species of Scenopinus Latreille (Diptera: Scenopinidae) from Australia
}

\author{
SHAUN L. WINTERTON \\ Entomology, Queensland Department of Primary industries \& Fisheries, 80 Meiers Road, Indooroopilly, Queensland, Australia 4068. \\ School of Integrated Biology, University of Queensland, St Lucia, Queensland, Australia 4072. \\ E-mail: shaun.winterton@dpi.qld.gov.au
}

Scenopinidae are a small family (ca. 300 species) of asiloid flies distributed on all continents except Antarctica. This family is closely related to Therevidae (Stiletto flies) based on the secondarily segmented characteristic of their larvae. Scenopinids are typically very small flies, frequently less than $5 \mathrm{~mm}$ total body length.

As with stiletto flies, the aridification of Australia has lead to significant diversification in Scenopinidae fauna with almost $25 \%$ of the world's described species (Kelsey 1973). Of those described species in Australia (ca. 80), all are endemic, with only two of the six described genera found outside of the region (Kelsey 1989). Like in all other biogeographic regions, the cosmopolitan Scenopinus Latreille is the dominant genus with 32 species described; this number will surely increase as more species are discovered.

The unusual crested frons of Scenopinus pallidipennis (Paramonov) prompted Paramonov (1955) to describe it as the monotypic genus Scenopinula Paramonov. The male was unknown at the time but Kelsey (1969) suspected that it was simply a highly derived species of Scenopinus. Kelsey (1975) subsequently described the male of the species, which did not have the crested frons and displayed all the characteristics of Scenopinus, to which Kelsey placed the species and thus synonymised Scenopinula. Closely related to Scenopinus pallidipennis, Scenopinus stephanos sp. nov. is described herein from a single female specimen from south-western Queensland. This species is distinctive from S. pallidipennis not only by the much larger body size and larger frontal callus in the female, but also by the grossly enlarged fore legs relative to the mid and hind legs.

\section{Taxonomy}

\section{Scenopinus stephanos sp. nov. (Fig. 1)}

Etymology. The specific epithet is derived from the Greek: stephanos; crown, diadem, referring to the crown-like frontal callus of the female.

Type material. Holotype female, AUSTRALIA: Queensland: Charleville, $5.5 \mathrm{~km}$ on road to Augathella; 5.iii.1989; E. Dahms, G. Sarnes; sweep. coccoid inf[ested] Aristida sp. Condition: good (glued on card). Type specimen (T.156204) is deposited in the Queensland Museum (QMBA) in Brisbane, Australia.

Diagnosis. Wing clear hyaline, venation brownish; frons with large, crown-like callus; halteres dark-brown; front legs much more robust than mid or hind legs; abdomen uniform black.

Description. Body length: female $3.0 \mathrm{~mm}$. Head: (Fig. 1) Glossy black, frons much wider than ocellar tubercle, overlain with short, fine white setae; large truncated callus above antenna projecting anteriorly; sides of callus wrinkled in texture, truncated surface verrucous with short tubercles, slightly cleft longitudinally; face smooth with sparse white setae; antennae projecting ventrally, brown with short pale setae on scape and pedicel; mouthparts reduced, but apparently not vestigial; ocellar tubercle flattened with sparse white setae; postocular ridge smooth, without macrosetae; fine white setae laterally on occiput and gena.

Thorax: Glossy dark brown-black; scutum sparsely overlain with elongate, fine white setae; pleuron mostly glabrous, anterior part greatly recessed to accommodate enlarged forelegs when raised against pleuron; legs with coxae and femora glossy dark-brown, tibia and tarsi light brown; pale setae on femora, longer on posterior surfaces; anterior legs 
distinctly shorter and more robust than mid and hind legs, fore-tarsal claws enlarged; wing hyaline, venation brown; haltere dark brown, knob with narrow pale stripe ventrally.

Abdomen: Glossy black, overlain with sparse pale setae, longer laterally and on terminalia. Genitalia not dissected; sternite 8 slightly emarginate laterally; lateral areas and intersegmental membrane pale.

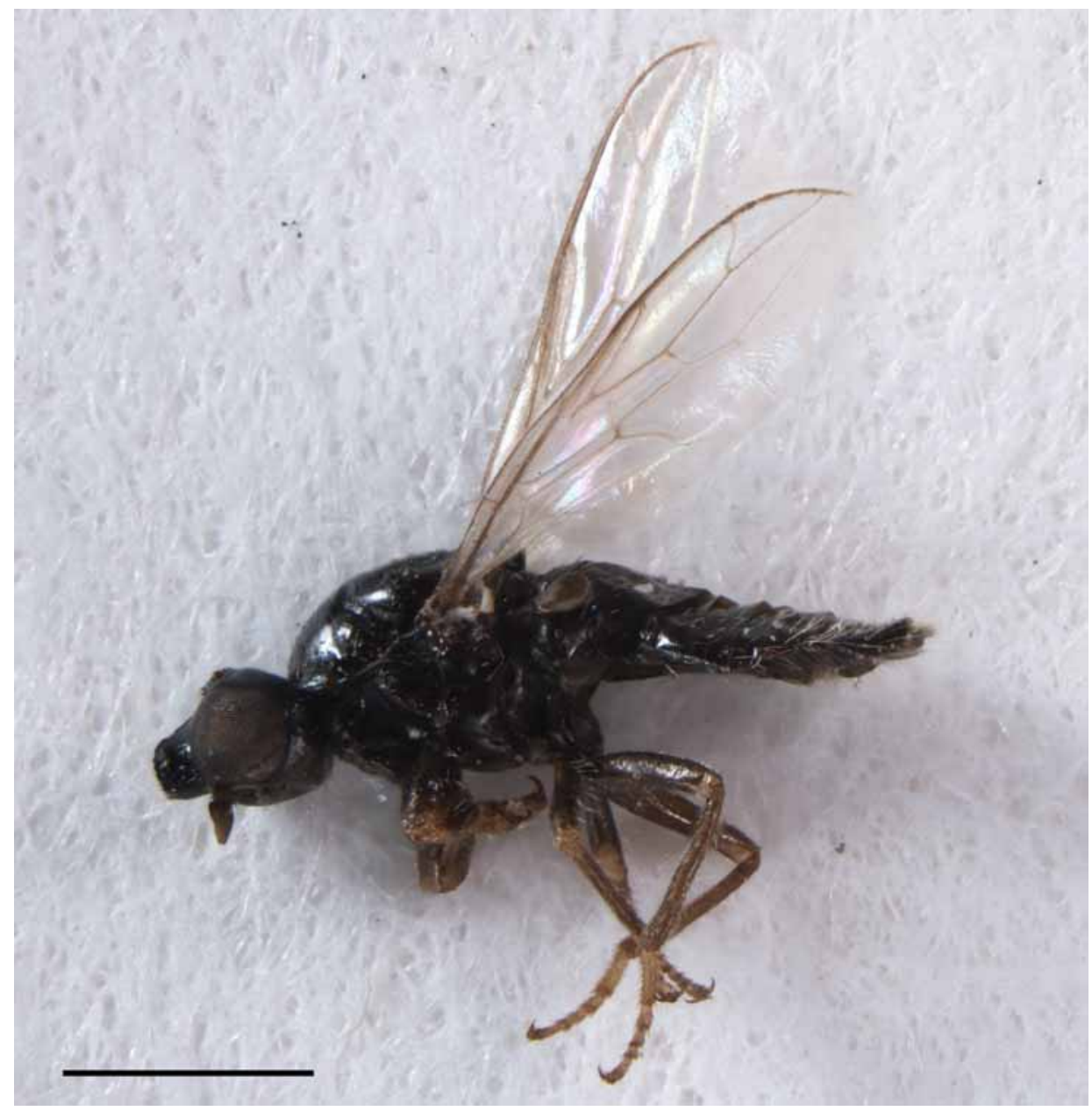

FIGURE 1: Scenopinus stephanos sp. nov. female type, lateral view. Scale line= $1.0 \mathrm{~mm}$.

Male. Unknown.

Comments. Scenopinus stephanos sp. nov. is distinct from all other Scenopinidae based on the large truncated frontal callus and the grossly enlarged forelegs (based on the female). It is placed in the genus Scenopinus based on the characteristic wing venation and antennal shape (see Kelsey 1969). The frontal callus is highly sclerotised and has a distinct verrucous longitudinal cleft. As the closely related $S$. pallidipennis male lacks the frontal callus of the female, it is also likely that the male of this new species also lacks a frontal callus. Scenopinus stephanos sp. nov. differs from $S$. pallidipennis by the larger size, darker wing venation, larger and differing shaped frontal callus, abdominal segments without white margins and enlarged fore legs. It appears that both S. stephanos sp. nov. and S. pallidipennis are inland species as all collecting records are from far inland sites in central Australia.

Acknowledgements. Thank you to Dr Chris Burwell (Queensland Museum) for loan of the Scenopinus specimen. This research was supported by a National Science Foundation (USA) grant (DEB-0614213) on Scenopinidae systematics. Statements and viewpoints expressed herein do not necessarily reflect the opinions of NSF.

\section{References}

Kelsey, L.P. (1969) A revision of the Scenopinidae (Diptera) of the world. Bulletin of the United States National Museum, 277, 1-336. 
Kelsey, L.P. (1970) New Scenopinidae (Diptera) from the Pacific area. Pacific Insects, 12, 39-48.

Kelsey, L.P. (1973) The zoogeographic distribution of known Scenopinidae (Diptera). Entomological News, 84, 329332.

Kelsey, L.P. (1975) New Australian Scenopinidae (Diptera). Journal of the Australian Entomological Society, 14, 197212.

Kelsey, L.P. (1989) Family Scenopinidae. In N.L. Evenhuis (ed.) Catalog of Diptera of the Australasian and Oceanian Regions. pp. 350-352. Bishop Museum Special Publication, 86, 1-1155.

Paramonov, S.J. (1955) A review of Australian Scenopinidae (Diptera). Australian Journal of Zoology, 1, $634-653$. 